\title{
Le soutien des étiages en Loire à l'aide de réservoirs situés dans le haut bassin. Application au barrage de Naussac
}

\author{
par J. Lefèvre \\ Ingénieur hydraulicien \\ à l'Agence Financière de Bassin \\ Loire-Bretagne
}

La Loire est un fleuve extrêmement irrégulier. En effet, sans parler de faits exceptionnels, il n'est pas rare, en Loire moyenne au niveau de Gien, de voir au cours d'une même année, le débit maximum 50 à 100 fois supérieur au débit minimum (respectivement $2470 \mathrm{~m}^{3} / \mathrm{s}$ et $25,5 \mathrm{~m}^{3} / \mathrm{s}$ en $1943,1850 \mathrm{~m}^{3} / \mathrm{s}$ et $25 \mathrm{~m}^{3} / \mathrm{s}$ en 1961 , etc.).

Ceoi montre la nécessité de «calmer» ce fleuve, donc à la fois d'écrêter ses crues dévastatrices (telles que celles de 1856,1866 ) et de soutenir ses étiages qui peuvent apporter une certaine gêne pour l'économie d'une région.

L'Agence Financière de Bassin Loire-Bretagne a mis au point un programme de soutien des étiages qui devrait se concréticer, dans un premier temps, par la mise en service du réservoir de Naussac. Dans l'avenir, d'autres réservoirs seront construits dans le même but ainsi que le barrage de Villerest, destiné à lutter aussi contre les crues de la Loire.

Cet article a pour objet de montrer le mode de gestion envisagé pour soutenir les étiages de l'Allier et de la Loire moyenne par le seul barrage de Naussac.

\section{Le modèle}

\section{Hypothèses}

Le soutien envisagé des étiages doit être tel que:

- l'objectif de débit à Gien soit le maximum possible;

- l'objectif de débit à Vieille-Brioude sur l'Allier doit être de 6 ou $8 \mathrm{~m}^{3} / \mathrm{s}$ si possible; ceci doit être fait:

/ en tenant compte du fait que la gestion de la retenue doit être compatible avec une exploitation touristique du plan d'eau pendant la saison estivale;

- / et en supposant que l'on dispose de la capacité maximum $\left(190 \mathrm{~m}^{3}\right)$ garantie par prélèvement gravitaire dans le Chapeauroux et pompage dans l'Allier.

\section{Type de modèle}

Au début des études, il fut envisagé un modèle déterministe dans lequel les prévisions de débit au jour $j$ aux points objectifs étaient faites en extrapolant la tendance constatée au jour $j-1$. Il s'agissait d'une extrapolation linéaire des logarithmes des $N$ derniers jours, et pendant les épisodes de crue, on employait une courbe de tarissement de la forme $q=q o e^{-a t}$.

Les calculs des lâchures n'étaient alors faits qu'après une critique de la prévision.

Le résultat de ce mode de gestion ne fut pas encourageant (gaspillage d'eau, manque d'eau en cas de variation importante de débits) et il fut donc décidé de s'en remettre à un modèle entièrement probabiliste où les résultats ne dépendraient que de ce modèle et non pas de l'interprétation de celui qui serait chargé de la gestion. Nous nous sommes donc tournés vers des prévisions faites à partir de corrélations multiples débits-pluies-température.

\section{Principe du modèle}

Au début de chaque mois de l'étiage considéré, on essaie de prévoir l'écoulement moyen de la période restant à courir jusqu'à la fin de l'étiage. Cette prévision est émise avec un niveau de sécurité de $95 \%$. Cette prévision de débit, comparée à la distribution des débits observés dans le passé, permet de déterminer la sévérité de l'étiage à venir (probabilité de non dépassement). Cette probabilité, associée au volume restant dans la retenue (donc théoriquement la déficience qu'il est possible de couvrir), permet de déterminer l'objectif de débit accessible à Gien pendant la période considérée.

Après avoir fixé cet objectif, il faut prévoir les lâchures qui seront faites journellement à partir de Naussac. Si le 
temps de transfert $\Delta T$ est connu entre le barrage et VieilleBrioude d'une part, Gien d'autre part, il ne reste qu'à émettre les prévisions de débit avec un décalage de temps égal à $\Delta T$ et compléter, s'il y a lieu, par des lâchures pour atteindre l'objectif de débit.

Exemple :

- au jour $J$ on prévoit pour le jour $J+\Delta T$ un débit $q(J+\Delta T)$

- la lâchure à effectuer sera donc $L=O D-q(J+\Delta T)$; $L \quad$ étant $\geqslant O$;

$O D$, l'objectif de débit déterminé auparavant.

\section{Choix des données utilisées pour émettre ces prévisions}

(voir figure 1)

\section{Variables débits}

Il était important d'avoir des séries assez longues. Dans la mesure où ceci était possible, et si la période était suffisamment représentative, cela évitait d'avoir à créer des séries de nombres au hasard, ce qui donne toujours un risque d'erreur.

Nous avons donc utilisé les stations de jaugeage suivantes:

- Gien, Villerest, Bas-en-Basset sur la Loire;

- Chadrac sur la Borne;

- Chatel-Montagne sur la Besbre;

- Vieille-Brioude sur l'Allier;

- Giroux sur la Dore;

- Besserve sur la Sioule;

et retenu, comme période d'étude, 1936-1969.

Les stations semblent assez représentatives et posséder une bonne stabilité.

\section{Variables climatologiques}

Nous avons choisi quinze postes aussi représentatifs que possible du bassin. Ce sont les postes de Fay-sur-Lignon, Le Monastier, Tarentaise, Viverols, Belvezet, Saint-Flour, Boisgrand, Egliseneuve-d'Entraigues, Clermont-les-Landais, Gelles, Hérisson, Fourneaux, Noiretable, Château-Chinon, Montceau-les-Mines.

En effet, la contribution des pluies aux écoulements d'été-automne ne provient pas seulement des hauts bassins de la Loire et de l'Allier, mais aussi du Forez et du Morvan.

La représentativité et la qualité des stations pluviométriques et thermométriques paraissent acceptables pour les buts de l'étude: leur choix a été effectué après un grand nombre d'essais parmi vingt-sept stations, lors d'une étude antérieure faite par E.D.F. sur les étiages de la Loire à Blois.

Au point de vue température, la température relevée à Clermont-les-Landais est considérée comme un témoin suffi. sant de la température de l'air sur le bassin concerné.

Pour l'utilisation de ces variables pluviométriques, nous avons été obligés de distinguer deux catégories :

- celles qui servent aux prévisions à long terme $(2,3$ ou 4 mois);
- celles qui servent aux prévisions à court terme (1 à 7 jours).

Les premières n'ont pas besoin d'être connues au jour le jour : seule la valeur mensuelle est nécessaire. Elle peut être connue dans les trois ou quatre jours suivant la fin d'un mois donné. A chacune des stations utilisées pour ce type de prévisions, seul un pluviomètre ou un pluviographe est nécessaire. Les quinze postes ont été retenus pour ces prévisions.

Quant aux secondes, la connaissance de la précipitation journalière est nécessaire. Il est donc nécessaire de prévoir en ces stations des pluviophones. Le coût de ces appareils et, bien entendu, le risque d'ennui sur les lignes téléphoniques en limite forcément le nombre.

Pour trouver ces postes nous avons donc été amenés à faire une étude dans laquelle nous avons fait intervenir les critères suivants :

- importance des précipitations;

- liaisons avec le débit de la Loire à Gien (pour différents échantillons de débit);

- les autocorrélations entre postes.

C'est ainsi que nous avons été amenés à sélectionner les postes suivants: Fay-sur-Lignon, Noiretable, Tarentaise, Saint-Flour, Château-Chinon et Gelles, ainsi que ClermontAulnat pour les températures. Ce choix a d'ailleurs pu être confirmé par une analyse factorielle des correspondances.

\section{Détermination des objectifs de débits Equations de prévisions à long ou moyen terme}

Au début de l'étiage, on doit déterminer la valeur de lobjectif de débit accessible à Gien durant la durée de l'étiage; au début de chacun des mois à venir il faut réajuster cette valeur, afin de tenir compte des écoulements qui se sont effectivement réalisés et, ainsi, serrer de plus près la réalité.

Il s'agit donc, dans un premier temps, de rechercher, à l'aide des observations hydroclimatologiques disponibles, la meilleure explication possible des apports de la Loire à Gien pendant une période de $k$ mois consécutifs, soit $E i j(k=i+j-1)$. Cette prévision se fait, d'une part avec un index le caractérisant les conditions initiales du bassin et, d'autre part, des index $P_{m}$ caractérisant les conditions météorologiques aléatoires qui surviendront pendant la période considérée: on obtient une équation de la forme:

$$
E i j=A c I e+B m P m+\varepsilon_{k}
$$

$\varepsilon_{k}$ étant le résidu non expliqué.

Comme il est possible de déterminer de façon précise la fonction de répartition (loi Gamma incomplète) du terme $P=B m P m$ (tout au moins des intervalles $1 \%-99 \%$ ), pour émettre une prévision à la date $i$ pour la période $(i, j)$, connaissant les $I e$, il suffit de déterminer la ou les probabilités utiles de l'événement $P$.

Dans le cas qui nous intéresse, ces prévisions ont été émises avec un niveau de sécurité de $95 \%$. En confrontant cette prévision d'apport avec la distribution empirique ou 
calculée des apports de 1936-1969, on peut en déduire la probabilité de non dépassement (donc la sévérité de l'étiage).

Il a été établi, d'autre part, la distribution des déficiences de la Loire à Gien en fonction de différents objectifs de débits.

Puisque l'on connait à tout instant le volume disponible en amont, et que l'on vient de calculer la probabilité de non dépassement des mois à venir, on en déduit l'objectif à respecter (voir fig. 2).

\section{Equations de prévision à court terme}

Avant toute chose, pour préciser ce court terme il faut essayer de déterminer le temps de transfert de ces lâchures.

\section{Temps de transfert des lâchures}

Il s'agit là d'un problème assez délicat à traiter puisqu'il semble a priori que :

- ce temps doit varier selon l'importance des lâchures;

- il varie aussi selon l'importance du débit en rivière lors de la lâchure;

- il n'est pas le même selon qu'il s'agisse d'un front d'onde se déplaçant ou simplement d'un transfert.

Comme on le voit, théoriquement, ce temps varie selon les conditions d'écoulement mais la gestion serait devenue absolument inextricable s'il avait fallu faire varier ce temps.

Nous avons donc fixé, une fois pour toutes, la durée de ces transferts. Pour ce, nous avons utilisé les coefficients d'autocorrélation croisés entre Gien et les différentes stations amont. Ceux-ci ont été établis à partir des débits du jour $j$ à Gien et des débits des jours $j, j-1, j-2, \ldots j-8$ aux stations en amont (Vieille-Brioude, Pont-du-Bouchet, Giroux, ...)

Nous avons obtenu les résultats suivants :

\begin{tabular}{|c|c|c|}
\hline Echantillon & Total & $\begin{array}{r}\text { Tronqué } \\
Q \leqslant 75 \mathrm{~m}^{3} / \mathrm{s}\end{array}$ \\
\hline Gien - Naussac $(470 \mathrm{~km}) \ldots \ldots \ldots$ & 5 jours & 7 jours \\
Gien - Vieille-Brioude $(360 \mathrm{~km}) \ldots$ & 4 jours & 6 jours \\
Gien - Pont-du-Bouchet $(290 \mathrm{~km})$. & 3 jours & 6 jours \\
Gien - Giroux $(240 \mathrm{~km}) \ldots \ldots \ldots$ & 3 jours & 6 jours \\
Gien - Chadrac $(460 \mathrm{~km}) \ldots \ldots \ldots$ & 5 jours & 6 jours \\
\hline
\end{tabular}

Nous avons done adopté comme décalages:

1 jour entre Naussac et Vieille-Brioude,

7 jours entre Naussac et Gien,

d'où les délais d'émission des prévisions.

\section{Equations de prévision}

Après avoir essayé différents groupements de variables, surtout en ce qui concerne les variables climatologiques, nous sommes arrivés aux résultats suivants.

\section{PLAN DE SITUATION}

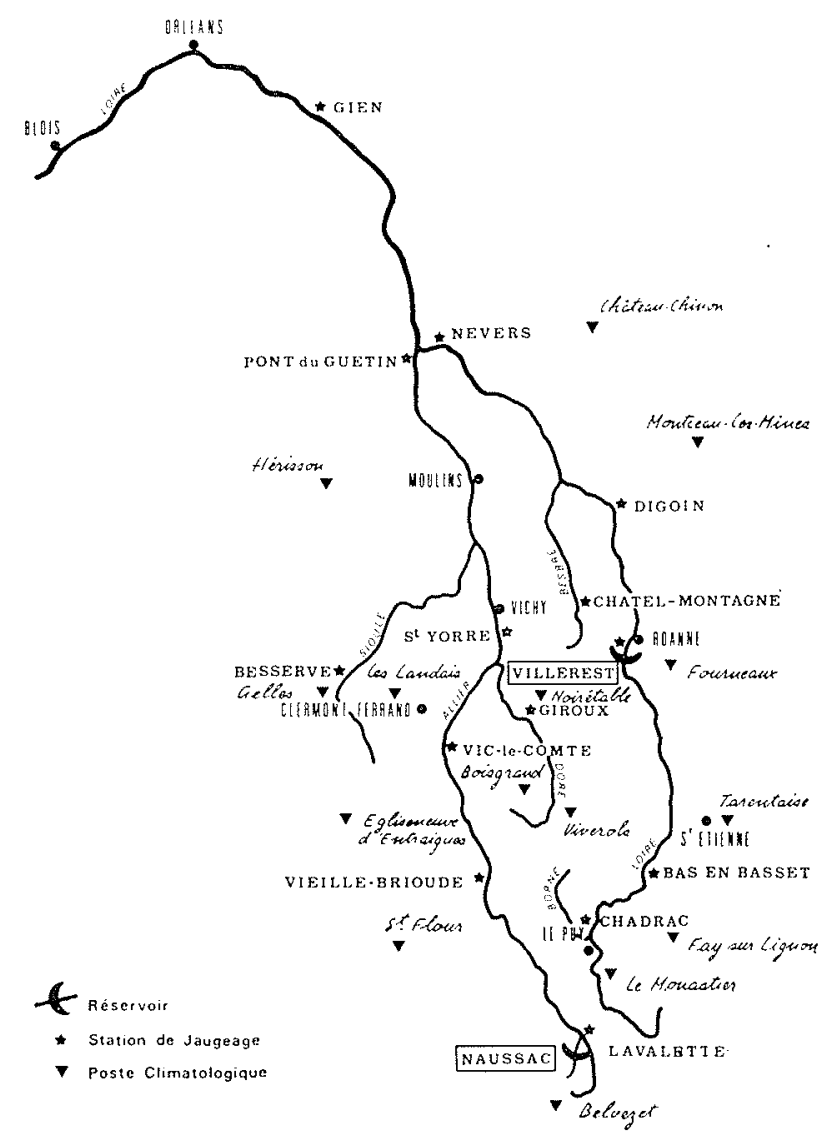

$1 /$
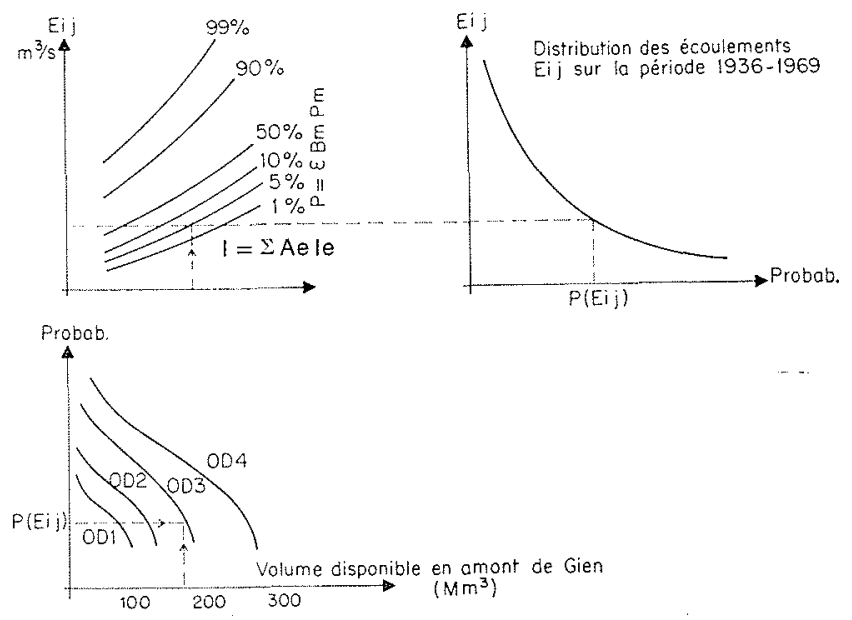

2/ Détermination des objectifs de débits 
Prévision du débit de l'Allier à Vieille-Brioude

(délai : 1 jour)

En fait, au début du jour $j$, on émet la prévision pour ce jour $j+1$, et on ne peut donc utiliser que les données du jour $j-1$ ou antérieures. Le délai réel est donc de 2 jours. $V B(j) \quad$ est le logarithme népérien du débit du jour $j$ à Vieille-Brioude;

$T M V B \quad$ la température moyenne des 4 derniers jours à Clermont-Aulnat;

IV $(j-1) \quad$ la somme des précipitations du jour $(j-1)$ aux postes de Noiretable, Tarentaise, Faysur-Lignon;

MIV $3(j-1)$ la moyenne des précipitations aux 3 postes précédents sur les jours $(j-1),(j-2)$ et $(j-3)$;

$I B(j-2) \quad$ la précipitation du jour $(j-2)$ à SaintFlour;

MID2 $(j-1)$ la moyenne des précipitations des jours $(j-1)$ et $(j-2)$ à Saint-Flour;

nous obtenons l'équation suivante :

$V B(j+1)=0,6255 V B(j-1)+0,2592 V B(j-3)$

$-0,0086 T M V B+0,006 I V(j-1)$

$-0,008 I B(j-2)+0,0262 M I D 2(j-1)$

$+0,0044 \operatorname{MIV} 3(j-1)+0,02293$

avec un coefficient de corrélation de 0,935 ; tous les coefficients sont significatifs au seuli de $99 \%$.

\section{Prévision du débit de la Loire à Gien}

(délai : 7 jours)

En utilisant les variables:

$G N(j) \quad$ logarithme népérien du débit du jour $j$ à Gien;

$P B(j) \quad$ logarithme népérien du débit du jour $j$ de la Sioule à Pont-du-Bouchet;

$G R(j) \quad$ logarithme népérien du débit du jour j de la Dore à Giroux;

$B B(j) \quad$ logarithme népérien du débit du jour $j$ de la Loire à Bas-en-Basset;

$V I(j) \quad$ logarithme népérien du débit du jour $j$ de la Loire à Villerest;

$T E(j-4) \quad$ la température du jour $(j-4)$ à ClermontAulnat;

$I G(j-1) \quad$ la somme des précipitations du jour $(j-1)$ mesurées aux 6 postes retenus;

$M I G 4(j-1)$ la moyenne de l'index $I G$ sur les 4 derniers jours de $(j-4)$ à $(j-1)$;

nous obtenons :

$$
\begin{aligned}
G N(j+7) & =0,5325 G N(j-1)-0,1992 G N(j-2) \\
& +0,1831 G N(j-4)+0,1673 P B(j-1) \\
& +0,0570 G R(j-1)+0,0865 B B(j-1) \\
& +0,0475 V I(j-1)-0,0105 T E(j-4) \\
& +0,0034 I G(j-1)+0,036 M I G 4(j-1) \\
& +1,5681 .
\end{aligned}
$$

Le coefficient de corrélation multiple est de 0,93 et tous les coefficients de régression sont significatifs à $99 \%$.

\section{Calcul des lâchures}

Une fois ces équations déterminées, iil ne reste plus qu'à effectuer le calcul des lâchures. Celui-ci s'effectue simplement.

Soit :

$O D B \quad$ le débit objectif à Vieille-Brioude;

$O D G \quad$ le débit objectif à Gien;

$Q B(j+1)$ le débit prévu au jour $(j+1)$ à VieilleBrioude;

$Q G(j+7)$ le débit prévu au jour $(j+7)$ à Gien;

la lâchure à effectuer devra satisfaire les deux objectifs de débit, même si elle amène un excédent en un des denx points. La lâchure sera donc de :

$L N=M A X[(O D B-Q B(j+1), O D G-Q G(j+7)]$

\section{Efficacité de la gestion}

Pour juger de l'efficacité de la gestion, nous avons défini un certain nombre de coefficients :

$K E=$ coefficient d'efficacité des lâchures:

$$
K E=-\frac{\text { volume lâché efficace }(E F F)}{\text { volume total des lâchures }(L A C H)}
$$

$E F F$ est le volume ayant effectivement contribué à combler tout ou partie de la déficience rapportée à l'objectif de débit choisi.

Comme il y a deux points de contrôle, EFF est égal au maximum des $E F F$ aux 2 points.

$K C=$ coefficient de couverture de la déficience:

$$
K C=\frac{\text { volume lâché efficace }(E F F)}{\text { volume total de la déficience }(D E F E)}
$$

La déficience est le volume qu'il faudrait combler pour atteindre l'objectif de débit.

$K U=$ coefficient d'utilisation des volumes stockés :

$$
K U=\frac{\text { volume total des lâchures }(L A C H)}{\text { volume disponible }(D I S P)}
$$

La partie de la déficience non couverte par les lâchures constitue le déficit (DEFT). Celui-ci, chaque jour est égal au maximum des DEFT aux points de contrôle. D'autre part, l'excédent $(E X C)$ est constitué par ce qui a été lâché en trop. Il est égal au minimum des $E X C$ aux points de contrôle.

\section{Simulation de la gestion - Résultats}

\section{Contrôle des volumes lâchés}

La simulation de la gestion a été effectuée sur la période 1936-1969, jour par jour sur toute la période d'étiage. Elle a, d'autre part, été effectuée pour un objectif de débit de l'Allier à Vic-le-Comte de $20 \mathrm{~m}^{3} / \mathrm{s}$ (ce qui correspond à $8 \mathrm{~m}^{3} / \mathrm{s}$ à Vieille-Brioude). 
La répartition mensuelle des lâchures s'effectue approximativement dans les proportions suivantes:

$$
\begin{aligned}
& 3 \% \text { en juin; } \\
& 16 \% \text { en juillet; } \\
& 33 \% \text { en août; } \\
& 31 \% \text { en septembre; } \\
& 14 \% \text { en octobre; } \\
& 3 \% \text { en novembre. }
\end{aligned}
$$

\begin{tabular}{|c|c|c|c|}
\hline & $K U$ & $K E$ & $K C$ \\
\hline Juin. . . . . . . & & 0,77 & 0,68 \\
\hline Juillet. . . . . . . & & 0,81 & 0,82 \\
\hline Août. . . . . . . & & 0,9 & 0,85 \\
\hline Septembre. . . . & & 0,91 & 0,82 \\
\hline Octobre...... & & 0,85 & 0,81 \\
\hline Novembre. . . . & & 0,77 & 0,87 \\
\hline TOTAL. . . & 0,45 & 0,89 & 0,83 \\
\hline
\end{tabular}

En moyenne : $K U=0,45 \quad K E=0,89 \quad K C=0,83$.

La déficience est donc couverte à $83 \%$ en moyenne et les lâchures se sont révélées efficaces à $89 \%$ en moyenne.

Mois par mois nous obtenons les résultats suivants :

L'efficacité des lâchures est maximale pendant les mois d'août et septembre, mois où la déficience elle-même est plus grande.

Il nous a semblé intéressant, d'autre part, de s'écarter des chiffres moyens pour analyser les résultats de la gestion dans certains cas particuliers. Nous avons donc découpé la chronique des années en un certain nombre d'échantillons :

- l'échantillon A caractérise les années les plus sèches: $1945,1947,1949,1950,1952,1962$;

- l'échantillon $\mathrm{B}$ correspond à la moitié sèche de la période

\begin{tabular}{|c|c|c|c|}
\hline & A & B & $\mathrm{C}$ \\
\hline$K U$ & 0,90 & 0,68 & 0,23 \\
\hline$K E$ & 0,94 & 0,91 & 0,81 \\
\hline$K C$ & 0,88 & 0,84 & 0,79 \\
\hline
\end{tabular}
1936-1969. Il est composé de A ainsi que des années $1942,1943,1946,1953,1955,1957,1959,1961,1964$, 1966, 1967;

- l'échantillon C représente lui la moitié humide.
Les résultats globaux par échantillon sont alors:

Il se confirme donc bien que plus l'étiage est sévère, meilleure est la gestion. Mais il faut bien noter que $75 \%$ des volumes lâchés le sont sur la moitié sèche, ce qui représente d'ailleurs exactement le même pourcentage pour la déficience.

En résumé, du point de vue couverture de la déficience, on peut estimer que ce type de gestion a été assez bon. Il ne pourrait être amélioré que par une amélioration des prévisions, ce qui semble assez difficile.

\section{Contrôle des débits}

L'amélioration est quand même assez sensible puisque, si l'on se réfère au débit du mois le plus sec de fréquence décennale, on obtient les résultats suivants :

\begin{tabular}{|l|c|c|c|}
\hline & $\begin{array}{c}Q 10 \\
\text { maturel } \\
\left(\mathrm{m}^{3} / \mathrm{s}\right)\end{array}$ & $\begin{array}{c}\text { Q10 } \\
\text { avec } \\
\text { réalimentation } \\
\left(\mathrm{m}^{3} / \mathrm{s}\right)\end{array}$ & RAPPORT \\
\cline { 1 - 2 } Blois. . . . . . & 40 & 52 & 1,3 \\
Gien. . . . . & 28 & 42 & 1,5 \\
Pont-du-Guétin. . & 16 & 30 & 1,9 \\
Saint-Yorre. . . & 9 & 22 & 2,4 \\
Vic-le-Comte. . . & 5,2 & 15 & 2,9 \\
Vieille-Brioude. . & 1,7 & 8,4 & 4,9 \\
Lavalette. . . . . & 0,5 & 2,5 & 5 \\
& & & \\
\hline
\end{tabular}

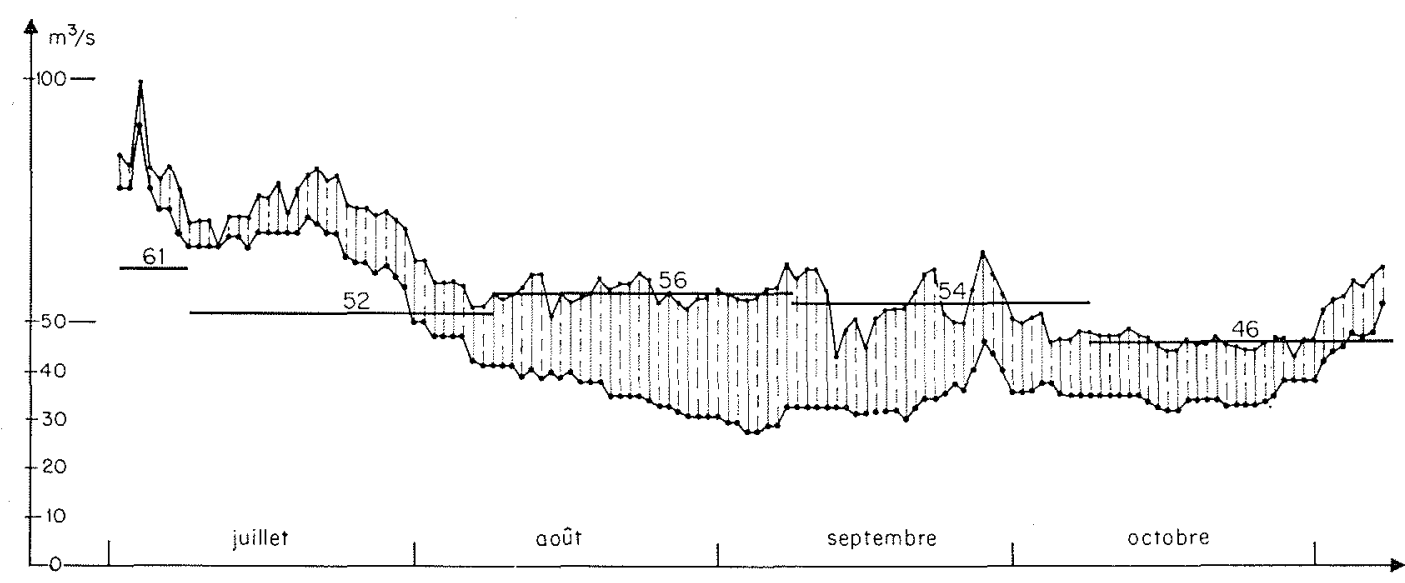

3/ Simulation de la gestion de Naussac. $2^{\circ}$ phase. Débits réels et induits de la Loire à Gien. Année 1962. 


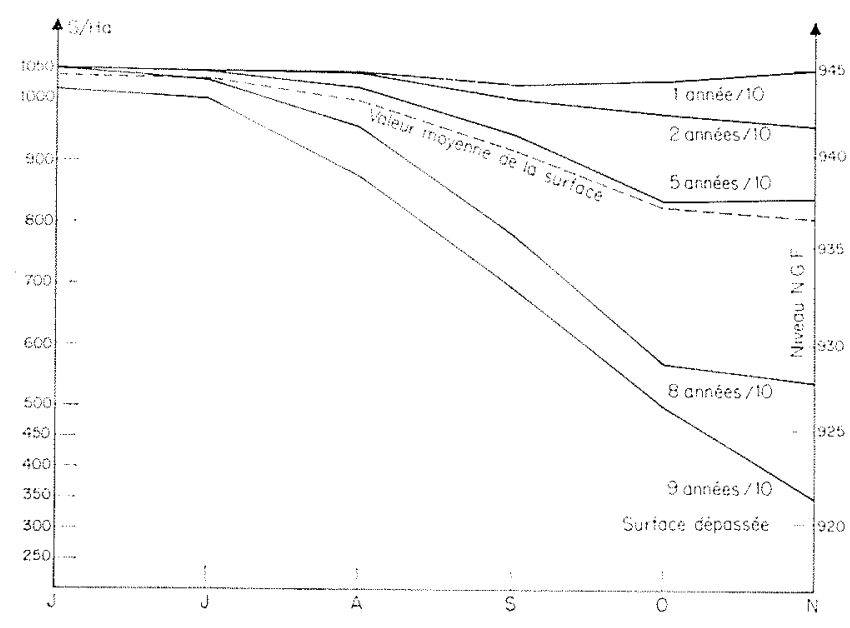

$4 /$ Simulation de la gestion de Naussac. $2^{\mathrm{e}}$ phase. surface de la retenue du $1^{\mathrm{er}}$ juin au $1^{\mathrm{r}}$ novembre.

On voit donc que l'augmentation de débit n'est pas négligeable du tout puisque, même à Blois, en cas d'années très sèches, on ajoute $30 \%$ du débit.

Le fait que l'objectif de débit à Vieille-Brioude soit respecté, et ne le soit pas à Vic-le-Comte, montre bien que l'on a procédé par corrélation entre les deux stations, n'ayant pas de données suffisantes à Vic-le-Comte.

Nous avons, d'autre part, tracé sur un graphique, les hydrogrammes réels et induits dans le cas d'une année assez sèche, par exemple 1962 (voir fig. 3).

$\mathrm{Si}$ une telle année se reproduisait, on pourrait espérer les améliorations suivantes:

$Q_{1}$ étant le débit mensuel minimum réel en 1962;

$Q_{2}$ étant le débit mensuel minimum s'il y avait eu réalimentation;

\begin{tabular}{|c|c|c|c|}
\hline & $\begin{array}{c}Q_{1} \\
\left(\mathrm{~m}^{3} / \mathrm{s}\right)\end{array}$ & $\underset{\left(\mathrm{m}^{3} / \mathrm{s}\right)}{Q_{:}}$ & $R=Q_{n} / Q$ \\
\hline Lavalette. . ... & 0,66 & 5,66 & 8,6 \\
\hline Vieille-Brioude. & 1,92 & 12,6 & 6,6 \\
\hline Vic-le-Comte. . & 6,8 & 18,3 & 2,7 \\
\hline Saint-Yorre. . . & 7,85 & 19,9 & 2,5 \\
\hline Pont-du-Guétin. & 12,8 & 28,3 & 2,2 \\
\hline Gien. . . . . . . & 34 & 46,2 & 1,4 \\
\hline Blois. . . . . . & 46 & 58,3 & 1,3 \\
\hline
\end{tabular}

On retrouve bien la même importance d'amélioration pour les années sèches : en Loire moyenne, on peut compter sur environ 12 à $13 \mathrm{~m}^{3} / \mathrm{s}$ en plus. Pour une année de ce type, on ajoute plus de $10 \mathrm{~m}^{3} / \mathrm{s}$ à partir de Vieille-Brioude.

\section{Contrôle de la cote ef de la surface du plan d'eau}

Du $1^{\text {rr }}$ juin au 31 octobre, nous avons calculé, de quinzaine en quinzaine, la valeur du volume restant dans la retenue, la cote et la surface du plan. Ceci est intéressant pour savoir si cette retenue peut être exploitée pendant la saison comme un plan d'eau touristique.

De ce point de vue seules la surface et la cote nous intéressent.

En admettant que la saison touristique ait principalement lieu en juillet et août, on peut constater qu'au $1^{\text {er }}$ septembre :

- 1 année sur 2, la superficie est supérieure à 940 ha (cote 941), c'est-à-dire que cela correspond à une diminution de $10 \%$ de sunface et $9 \%$ de cote;

- 1 année sur 10 seulement, la surface est inférieure à 700 ha (diminution de $30 \%$ ), ce qui correspond à la cote 933 (soit un marnage de $12 \mathrm{~m}$ ).

D'après la simulation, la superficie moyenne du plan d'eau est :

\begin{tabular}{|c|c|}
\hline Date & $\underset{\text { (ha) }}{S}$ \\
\hline $1^{\text {er }}$ juin. . . . . . . . . . & 1050 \\
\hline $1^{\text {er }}$ juillet. . . . . . . . . & 1050 \\
\hline $1^{\text {er }}$ août. $\ldots \ldots \ldots \ldots$ & 1020 \\
\hline $1^{\text {er }}$ septembre. . . . . . . & 940 \\
\hline $1^{\text {er }}$ octobre. . . . . . . & 835 \\
\hline
\end{tabular}

Cette faible variation semble donc compatible avec une utilisation touristique de la retenue (voir fig. 4).

\section{Aperçu sur \\ la future gestion des barrages de Naussac et Villerest}

Après Naussac, le second barrage qui devrait être construit est celui de Villerest. Nous avons commencé à étudier la gestion simultanée de ces deux barrages et le problème ici se complique.

Il faut en effet concilier :

- la fonction d'écrêtement des crues d'automne et de printemps à Villerest, donc une cote maximale à respecter en juin et surtout en septembre;

- la fonction de soutien des étiages des deux barrages devant permettre de respecter trois objectifs de débit - l'un sur l'Allier, l'autre sur la Loire supérieure (à garantir en année cinquantenaire) et le troisième à Gien en Loire moyenne;

- l'utilisation touristique de Naussac.

I] faut, d'autre part, tenir compte du fait que les temps de transfert des lâchures entre Gien et Naussac d'une part, Gien et Villerest d'autre part, ne sont pas les mêmes ( 7 jours et 5 jours).

Ceci nous a amené à adopter comme principes

- Villerest est vidangé en premier; 
- Dans la première partie de l'étiage, Naussac (barrage auxiliaire) assume seulement l'objectif de débit sur l'Allier, Villerest (barrage pilote) les deux autres sur la Loire; par la suite c'est l'inverse : Villerest (auxiliaire) assure l'objectif sur la Loire supérieure et Naussac (pilote) les deux autres.

Ces hypothèses nous ont permis de concilier les contraintes qui nous étaient imposées (hormis le fait d'être obligé en année humide de descendre à une certaine cote au 15 septembre pour recevoir les crues d'automne, ce qui nous a conduits à faire des lâchures «inutiles »).

Il a été intéressant de constater que les résultats de la gestion sont similaires : $88 \%$ de la déficience a été couverte en année sèche.

En Loire moyenne, les débits sont encore augmentés d'environ $10 \mathrm{~m}^{3} / \mathrm{s}$. Par contre, cette sorte de gestion fait apparaître des résultats moins bons sur l'Allier lorsque Villerest est le barrage pilote. En effet, l'Allier ne «profite» pas alors des lâchures importantes qui assurent l'objectif de Gien. Ceci devrait donc nous conduire à relever les objectifs de débit intermédiaire.

\section{Conclusion}

Avec, en plus, l'implantation du barrage de Serre-de-laFare, il ne devrait plus y avoir de problèmes d'eau depuis le haut bassin jusqu'en Basse Loire pratiquement. La réalimentation de la Loire aura donc un double but:

- assurer l'alimentation en eau des villes, industries et agricultures et éviter aussi la construction de certains petits barrages projetés à des prix de revient par $\mathrm{m}^{3}$ très élevés;

- améliorer par dilution la qualité de l'eau des axes réalimentés:

- garantir un volume de sécurité utilisable en cas de pollution accidentelle sur l'axe réalimenté.

\section{Références}

«Prévisions d'écoulements de la Loire à Gien pendant l'été ». $E D F-D T G$.

A. Valadou. - «Simulation de gestion de la retenue de Naussac (1 ${ }^{\text {re }}$ phase) 》. Agence de Bassin Loire-Bretagne (ETU/14).

J. LefÈvre et A. VAlAdou. - «Essai de gestion simultanée des barrages de Naussac et Villerest ». Agence de Bassin LoireBretagne (ETU/23).

\section{Discussion}

Président : M. BONNEvioI

M. le Président remercie M. Lefevre de son exposé très documenté sur un sujet en p'eine actualité et ouvre la discussion.

M. Carlier pose les deux questions suivantes :

Compte tenu des caractéristiques topographiques du site de Naussac, le marnage de quatre mètres indiqué par le conférencier me parât difficilement compatible avec l'utilisation de la retenue pour la pratique des sports nautiques.

Je souhaiterais savoir si on a pensé à la solution classique d'un barrage disposé à l'intérieur de la retenue et permettant de constituer un plan d'eau à niveau constant facilitant la pratique des sports nautiques.

Par ailleurs, le conférencier a signalé que la retenue de Naussac serait remplie partiellement par pompage dans l'A'lier; une station de pompage est prévue, à cet effet, à la Valette, au confluent du Donozeau et de l'Allier.

Je souhaiterais savoir si cette station sera concue pour pouvoir également turbiner les lâchures du barrage de Naussac, ce qui pourrait présenter un intérêt économique supplémentaire.

M. LEFEVRe répond par l'affirmative sur les deux points cidessus. Un plan d'eau à niveau constant d'une dizaine d'hectares sera aménagé dans la retenue de Naussac - qui s'étend sur un millier d'hectares - pour la pratique des sports nautiques. D'autre part, pour l'alimentation du réservoir de Naussac à partir de l'Allier, il est bien prévu une station de «pompage-turbinage».

M. Bernier demande des précisions sur les points ci-après :

$1^{\circ}$ Dans ce genre d'étude, on se fixe généralement un objectif sous la forme par exemple d'un débit minimum à assurer en un point critique du cours d'eau. Que! était dans le cas présenté, l'objectif choisi ?

$2^{\circ}$ A-t-on tenu compte des pertes d'eau au cours du transfert des débits entre le réservoir et Gien?

$3^{\circ}$ Le temps de propagation des éclusées entre le réservoir et Gien - on a admis sept jours - parait relativement faible. A-t-on fait des mesures?

\section{LEFEVRE répond :}

$1^{\circ}$ La Mission de Bassin «Loire-Bretagne 》 a défini comme objectif à moyen terme le maintien d'un débit au moins égal à $70 \mathrm{~m}^{3} / \mathrm{s}$ à Gien et à $100 \mathrm{~m} 3 / \mathrm{s}$ à Nantes. Mais cet objectif doit être atteint par la construction de trois barrages-réservoirs dans le HautBassin de la Loire; Naussac est le premier de ces trois ouvrages nous nous sommes bornés à rechercher par son action le plus fort débit possible à Gien ;

$2^{\circ}$ Dans l'état actuel des études, il n'a pas été tenu compte explicitement des «pertes». Aucune mesure n'a été faite à ce sujet et de toute façon se révèle difficile car la nature des terrains traversés varie énormément.

$3^{\circ}$ Des calculs d'auto-corrélation ont donné, pour le temps de propagation des débits entre Naussac et Gien, des va'eurs comprises entre six et huit jours. Des calculs hydrauliques, tenant compte des pentes et des vitesses moyennes dans les différents secteurs et pour divers état des eaux, donnent des chiffres variant de quatre à douze jours. Nous comptons préciser ce point par des essais et mesures sur le terrain dès que le réservoir de Naussac sera mis en eal.

A propos du point 2 ci-dessus, M. le Président précise :

Le réseau hydraulique de la Loire est fort développé et très complexe surtout en basses eaux. Les lâchures du réservoir de Naussac n'auront ni la brutalité, ni l'ampleur des crues naturelles et il n'est pas sûr que les estimations effectuées à partir d'observations tirées de celles-ci soient pour le moment très fiables.

M. Roche se penche sur les raisons qui ont rendu peu satisfaisant les modèles déterministes dont $M$. LeFEvre a signalé l'emploi au début de son exposé. Ces modè'es, dit-il, étaient trop schématiques pour représenter, par exemple, le processus de tarissement du cours d'eau; peut-être eût-il fallu introduire le régime des pluies à caractère a'éatoire pendant la période considérée.

C'est aussi l'avis de M. Lefevre, mais les études se sont rapidement concentrées sur un modèle probabiliste et le modèle déterministe a été abandonné. 
M. Durousseau apporte une intéressante observation sur l'importance des pertes constatées au cours des «lâchures » faites sur un cours d'eau à partir d'un réservoir d'amont.

Dans le hant bassin de l'Helpe majeure, affuent de la Sambre, dit-il, E.D.F. a construit un barrage-réservoir pour améliorer en étiage l'alimentation d'une centrale thermique, implantée au voisinage de Maubeuge. Sur le parcours de $50 \mathrm{~km}$ environ entre le réservoir et la centrale, des essais effectués récemment ont montré que, pour des lâchures de $30000 \mathrm{~m}^{3}$ par jour en période d'étiage sévère, la perte d'eau était de l'ordre de $20 \%$ du volume ci-dessus. Ce résultat n'est évidemment pas directement transposable au barrage de Naussac mais il incline le projeteur à la prudence.

En effet, observe M. le Président, les échanges entre les cours d'eau et leur nappe alluviale sont toujours des cas d'espèce. A l'occasion. d'un captage effectué vers Sancerre-Châtillon-sur-Loire sur les rives du fleuve - en terrain très perméable - nous avons constaté que le lit de ce demier était bien plus colmaté qu'on ne le pensait. La pollution grandissante tend d'ailleurs à accélérer ce colmatage.

Y a-t-il une explication au fait que les lâchures sont plus efficaces pendant les étiages sévères que pendant les étiages moyens, demande M. Durousseau ?

Cela résulte, répond $M$. Lefevre, de la meilleure définition des courbes de régression en étiage sévère du fait que la courbe de tarissement comporte moins de ces irrégularités qu'engendrent les pluies locales.

M. Tixeront intervient ensuite en ces termes:

Certaines données admises en ce qui concerne les délais de trans- mission des lâchures de Naussac à Gien et les coefficients d'efficacité qui en résultent semblent un peu optimistes. Puisqu'on doit établi les deux barrages de Naussac et Villerest, a-t-on étudié l'utilisation de Villerest en conjugaison avec celui de Naussac? Quel degré d'intérêt présente l'aménagement d'un bassin de compensation sur l'Allier près de son confluent avec la Loirc?

\section{Lefevre répond}

Les coefficients d'efficacité, dutilisation et de couverture de la déficience sont aussi bons sinon meilleurs lorsqu'on adjoint Villerest à la retenue de Naussac. Ceci est dû au fait qu'il n'y a que cing jours de transfert entre Vil'erest et Naussac (au lieu de sept à partir de Naussac). Un bassin de compensation sur l'Allier serait très intéressant (Le Veurdre par exemple) car il permettrait de rectifier avec un système de précision à un on deux jours (temps de transfert entre Nevers et Gien) les erreurs de prévision faites avec un délai de sept jours et de corriger éventuellement ce temps de transfert adopté quelque soit le débit nature! de l'étiage et le volume de la lâchure.

M. le Président confirme qu'il est envisagé d'implanter un barrage près du confluent de la Loire et de l'Allier au voisinage de SaintPierre-Le-Moutier. Les éludes ne sont pas encore assez avancées pour se faire une idée suffisante de l'intérêt économique de ce réservoir de compensation qui permettrait de moduler d'une façon plus fine - en été - les lâchures du réservoir de Naussac.

M. le Président clôt la discussion et donne la parole à M. LoHou pour l'exposé de la communication qu'il a établie en liaison avec M. SORMAYL

\section{Abstract}

\section{Upper valley reservoirs to maintain adequate low-water discharge in the river Loire The Naussac river barrage}

The river Loire has a very irregular regimen which sometimes results in an extreme'y low water level in the river. To remedy the situation the Loire-Brittany Development Office has produc ed a low-water discharge make-up programme, the first link of which is the Naussac barrage on the river Allier, a tributary of the river Loire. The investigation model allows for the following requirements:

1. Discharge of $8 \mathrm{~m}^{3 /} / \mathrm{s}$ at Vieille Brioude, $110 \mathrm{~km}$ down-river from the reservoir.

2. Highest possible discharge at Gien, $470 \mathrm{~km}$ from Naussac.

3. Reservoir management and operation to be consistent with development of tourism.

4. Reservoir to be built for full storage requirement $\left(190 \mathrm{Mm}^{2}\right)$ right from the start and to be filled by diverting flow unde gravity from the river Chapeauroux and pumping supplies from the river Allier.

At the beginning of each month of the considered low-wate period an attempt is made to predict the mean flow for the rest of the period, allowing for a $95 \%$ security level. By comparing the predicted figure with observed past discharge distribution the severity of future low-water conditions can be predicted (probability that these will not be exceeded)
From this probability and the amount of storage remaining in the reservoir (i.e. the theoretical deficiency that can be covered), an "available discharge" target can be established for Gien for the considered period (see method described in \$3)

A short-term forecast is then made, taking the transfer time of supplies released, from which water re'ease requirements are calculated. Transfer times are calculated by cross-autocorrelation.

Data from fifteen climatological and six river-gauging station were used for these forecasts.

Three coefficients were calculated to check the results: 1. a "release effectiveness" coefficient $(K E)$; 2. a "deficiency coverage" coefficient $(K C)$; and 3 . a "storage utilisation" coefficient $(K U)$.

The model simulation covered the period from 1936 to 1969. Its results can be considered satisfactory, since it was found that deficiency coverage averaged $83 \%$ and that $89 \%$ of the releases were eflective.

A brief description is also given of how the scheme will operate when the second link (the Villerest barrage on the Upper Loirc) becomes operational. In addition to sustaining adequate low-water discharge, this barrage will also provide effective flood control 